\title{
Receptor FGFRL1 acts as a tumor suppressor in nude mice when overexpressed in HEK 293 Tet-On cells
}

\author{
LEI ZHUANG $^{1}$, FLORIAN STEINBERG ${ }^{1,2}$ and BEAT TRUEB ${ }^{1,3}$ \\ ${ }^{1}$ Department of Clinical Research, University of Bern, CH-3008 Bern, Switzerland; \\ ${ }^{2}$ Center for Biological Systems Analysis, University of Freiburg, D-79104 Freiburg, Germany; \\ ${ }^{3}$ Department of Rheumatology, University Hospital, CH-3010 Bern, Switzerland
}

Received May 26, 2016; Accepted August 26, 2016

DOI: $10.3892 / 01.2016 .5245$

\begin{abstract}
Fibroblast growth factor receptor-like 1 (FGFRL1) is a transmembrane receptor that interacts with heparin and FGF ligands. In contrast to the classical FGF receptors, FGFR1 to FGFR4, it does not appear to affect cell growth and proliferation. In the present study, an inducible gene expression system was utilized in combination with a xenograft tumor model to investigate the effects of FGFRL1 on cell adhesion and tumor formation. It was determined that recombinant FGFRL1 promotes the adhesion of HEK 293 Tet-On ${ }^{\circledR}$ cells in vitro. Moreover, when such cells are induced to express FGFRL1 $\Delta \mathrm{C}$ they aggregate into huge clusters. If injected into nude mice, the cells form large tumors. Notably, this tumor growth is completely inhibited when the expression of FGFRL1 is induced. The forced expression of FGFRL1 in the tumor tissue may restore contact inhibition, thereby preventing growth of the cells in nude mice. The results of the present study demonstrate that FGFRL1 acts as a tumor suppressor similar to numerous other cell adhesion proteins. It is therefore likely that FGFRL1 functions as a regular cell-cell adhesion protein.
\end{abstract}

\section{Introduction}

Cell adhesion proteins form connections between individual cells and mediate the interactions of cells with the surrounding extracellular matrix (ECM) (1). In this way, they participate in various cellular functions, including signal transduction, communication, embryogenesis, inflammation and apoptosis.

The majority of cell adhesion proteins can be grouped into one of five families: The immunoglobulin family, the

Correspondence to: Professor Beat Trueb, Department of Clinical Research, University of Bern, Murtenstrasse 35, CH-3008 Bern, Switzerland

E-mail: beat.trueb@dkf.unibe.ch

Abbreviations: FGF, fibroblast growth factor; FGFR, fibroblast growth factor receptor; FGFRL1, FGFR-like protein 1

Key words: FGF, FGFR, FGFRL1, cell adhesion, tumor suppressor integrins, the cadherins, the selectins and the syndecans (1-3). Members of the immunoglobulin family promote cell adhesion in a calcium-independent manner. These proteins contain an extracellular section with a variable number of immunoglobin (Ig)-like domains, a relatively short intracellular domain and a single transmembrane domain. For example, cell adhesion molecule-1 (CADM1; also known as nectin-like protein 2) contains three extracellular Ig-like domains and binds with its intracellular domain to the adapter protein DAL1, which in turn anchors CADM1 to the actin cytoskeleton (1-3). Integrins are involved in the integration of the ECM with the cytoskeleton. These proteins represent noncovalently linked heterodimers composed of an $\alpha$ and a $\beta$ subunit. The majority of integrins acts as receptors for ECM proteins, including fibronectin, vitronectin, collagens and laminin, and recognizes the Arg-Gly-Asp sequence within target proteins (1-3). Cadherins are calcium-dependent cell adhesion molecules. E-cadherin, for example, contains an extracellular domain with 5 cadherin repeats, an intracellular domain and a transmembrane domain. The intracellular domain interacts with catenin and binds to the actin cytoskeleton (1-3). Selectins mediate the interactions between leukocytes and endothelial cells. They are composed of a single transmembrane domain, a short intracellular domain and an extracellular domain with a variable number of sushi motifs. Typically, selectins assist with the homing of lymphocytes to the lymph node (1-3). Finally, syndecans are proteoglycans located on the cell membrane. They contain a variable number of glycosaminoglycan chains attached to strategic serine residues of the polypeptide chain. In addition to mediating cell-matrix and cell-cell interactions, syndecans contribute to modulating the activity of heparin binding growth factors (1-3).

Evidence from previous studies indicates that cell adhesion proteins participate in tumor formation and metastasis $(2,3)$. Indeed, the progression of cancer is often associated with the loss of at least one cell adhesion protein. However, cell adhesion proteins can also function as tumor suppressors when overexpressed in tumor tissues $(2,3)$. It appears that their forced expression restores contact inhibition, a phenomenon observed in normal cells, but not in the majority of malignant cells. E-cadherin, for example, is often lost in tumors of epithelial origin and it has been demonstrated that the disruption of cell adhesion mediated by E-cadherin is associated with the development and progression of cancer (4). The 
forced expression of E-cadherin in tumor cell lines slows down cell proliferation and reduces cell invasiveness. By contrast, inhibiting E-cadherin using antibodies or antisense RNA restores the invasiveness of the cells (4). Likewise, integrin $\alpha 7$ is frequently mutated in human malignancies and such mutations are associated with cancer recurrence (5). It has been demonstrated that the increased expression of integrin $\alpha 7$ in leiomyosarcoma cells resulted in the reduction of colony formation. Moreover, increasing the expression of $\alpha 7$ integrin in mice with xenografted tumors inhibited tumor growth (5). Likewise, CADM1 has been implicated in cancer progression. Downregulation of CADM1 synthesis has been observed in a variety of human tumors, including breast cancer and esophageal squamous cell carcinoma $(6,7)$. However, it has been demonstrated that restoring CADM1 expression suppresses cell growth and slows down tumor invasion (7). It has therefore been concluded that the majority of the cell adhesion proteins can function as tumor suppressors $(2,3)$.

A previous study by our group described a novel transmembrane protein that resembles CADM1 $(8,9)$. This protein contains three extracellular Ig-like domains, a relatively short intracellular domain and a single transmembrane domain. It interacts with heparin and fibroblast growth factors (FGFs) in a manner similar to the classical FGF receptors (FRFRs), FGFR1-FGFR4 (10,11). For this reason, the novel protein was termed FGFR-like 1 protein (FGFRL1). However, FGFRL1 does not possess the intracellular tyrosine kinase domain required for signal transduction by transphosphorylation and consequently cannot mediate FGF signaling by itself.

The function of the novel receptor is currently unclear. Knockout mice with a targeted disruption of the FGFRL1 gene present a striking phenotype; they lack metanephric kidneys (12) and die at birth due to a weak, malformed diaphragm that cannot inflate the lungs after birth $(13,14)$. Cell culture experiments have demonstrated that FGFRL1 can act as a typical cell adhesion protein when coated on plastic dishes. FGFRL1 forms heterophilic interactions with heparan sulfate proteoglycans, such as glypican, at the cell surface of neighboring cells (15). A tetracycline-inducible system has been utilized, in which the expression of FGFRL1 could be controlled by the addition of the inducer doxycycline (16). It was observed that in the presence of doxycycline, cells aggregated and formed huge clusters, whereas in its absence, they tended to remain as individual clones (17).

In the present study, one of the tetracycline-inducible cell clones was characterized in more detail. The primary aim of the study was to determine whether FGFRL1 functions as a tumor suppressor in a manner similar to other cell adhesion proteins.

\section{Materials and methods}

Cell culture. The generation of tetracycline-inducible cell clones has been described in detail in a recent publication (17). The stable clone $\mathrm{K} 13 \Delta \mathrm{C}$ was produced by transfection of a cDNA for truncated FGFRL1 (corresponding to amino acid residues 1-417) into HEK 293 Tet-On ${ }^{\circledR}$ cells (Clontech Laboratories, Takara Bio Europe SAS, Saint-Germain-en-Laye, France). The cells were cultivated in an atmosphere of 5\% $\mathrm{CO}_{2}$ in Dulbecco's modified Eagle's medium (DMEM) supplemented with $10 \%$ fetal bovine serum, $100 \mathrm{U} / \mathrm{ml}$ penicillin and $100 \mu \mathrm{g} / \mathrm{ml}$ streptomycin (all Sigma-Aldrich, Buchs, Switzerland). To maintain the selective pressure for stable transfection, $100 \mu \mathrm{g} / \mathrm{ml}$ Hygromycin B (InvivoGen, San Diego, CA, USA) was used. Expression of FGFRL1 from the tetracycline responsive promoter was induced by the addition of $1 \mu \mathrm{g} / \mathrm{ml}$ doxycycline.

Adhesion experiments. Recombinant FGFRL1 protein was isolated from the conditioned media of HEK 293 cells that had been stably transfected with a cDNA for human FGFRL1 (corresponding to amino acid residues 1-357), as previously described (18). The recombinant protein was purified by chromatography on a column of Heparin Sepharose ${ }^{\circledR} 6$ Fast Flow (GE Healthcare Bio-Sciences, Pittsburgh, PA, USA) as previously described (18). FGFRL1 protein or bovine serum albumin (BSA; Sigma-Aldrich), which served as a control, was diluted to $20 \mu \mathrm{g} / \mathrm{ml}$ in phosphate-buffered saline (PBS) and droplets of the solution $(15 \mu \mathrm{l})$ were spotted onto $35-\mathrm{mm}$ petri dishes (non-tissue culture; catalog no. 82.1135; Sarstedt Co., Nümbrecht, Germany). Following incubation in a humidified chamber at $4^{\circ} \mathrm{C}$ for $16 \mathrm{~h}$, the solution was carefully aspirated and all residual sites of the petri dish were blocked with $1 \%$ BSA in PBS. Cells were seeded onto pre-coated petri dishes in serum-free medium $\left(2 \times 10^{6}\right.$ cells/plate $)$ and incubated for $1 \mathrm{~h}$ at $37^{\circ} \mathrm{C}$. Non-adherent cells were carefully removed by washing with PBS. Adherent cells were fixed with $4 \%$ paraformaldehyde (Sigma-Aldrich) and inspected under a microscope (Nikon Eclipse E800; Nikon AG, Zurich, Switzerland).

In a further experiment, the cells were cultivated in complete growth medium (as aforementioned) on uncoated petri dishes (non-tissue culture) for 1-3 days, and cell adhesion and cell-cell clustering were documented with a Zeiss Axiovert 10 microscope (Carl Zeiss AG, Oberkochen, Germany).

Immunocytochemistry. Cells grown on coverslips were washed with ice-cold PBS, fixed with $4 \%$ paraformaldehyde and permeabilized with $0.2 \%$ Triton X-100 (Sigma-Aldrich) in PBS. Non-specific sites were blocked with 3\% BSA in PBS. The fixed cells were incubated for $3 \mathrm{~h}$ at room temperature with a humanized monoclonal antibody $(1 \mu \mathrm{g} / \mathrm{ml})$ against FGFRL1 that had been prepared by our group in a previous study (19). Following three steps of washing with PBS, bound antibodies were detected with Cy2-labeled secondary antibodies (catalog no. 109-225-097; dilution 1:200; Jackson ImmunoResearch Laboratories, West Grove, PA, USA). The nuclei of the cells were stained with $1 \mu \mathrm{g} / \mathrm{ml}$ 4',6-diamidino-2-phenylindole (Invitrogen; Thermo Fisher Scientific, Inc., Waltham, MA, USA). To detect filamentous actin, fixed cells were treated in a similar way with tetramethylrhodamine-labeled phalloidin (Sigma-Aldrich).

Electron microscopy. Cells grown on cover slips were fixed with $2.5 \%$ glutaraldehyde in $30 \mathrm{mM}$ potassium phosphate buffer. The fixed cells were dehydrated in ethanol, critical point dried and sputter-coated with gold, as previously described (20). Finally, the specimens were inspected with a Philips XL 30 FEG scanning electron microscope operated at 10 kV (Philips, Amsterdam, The Netherlands). 

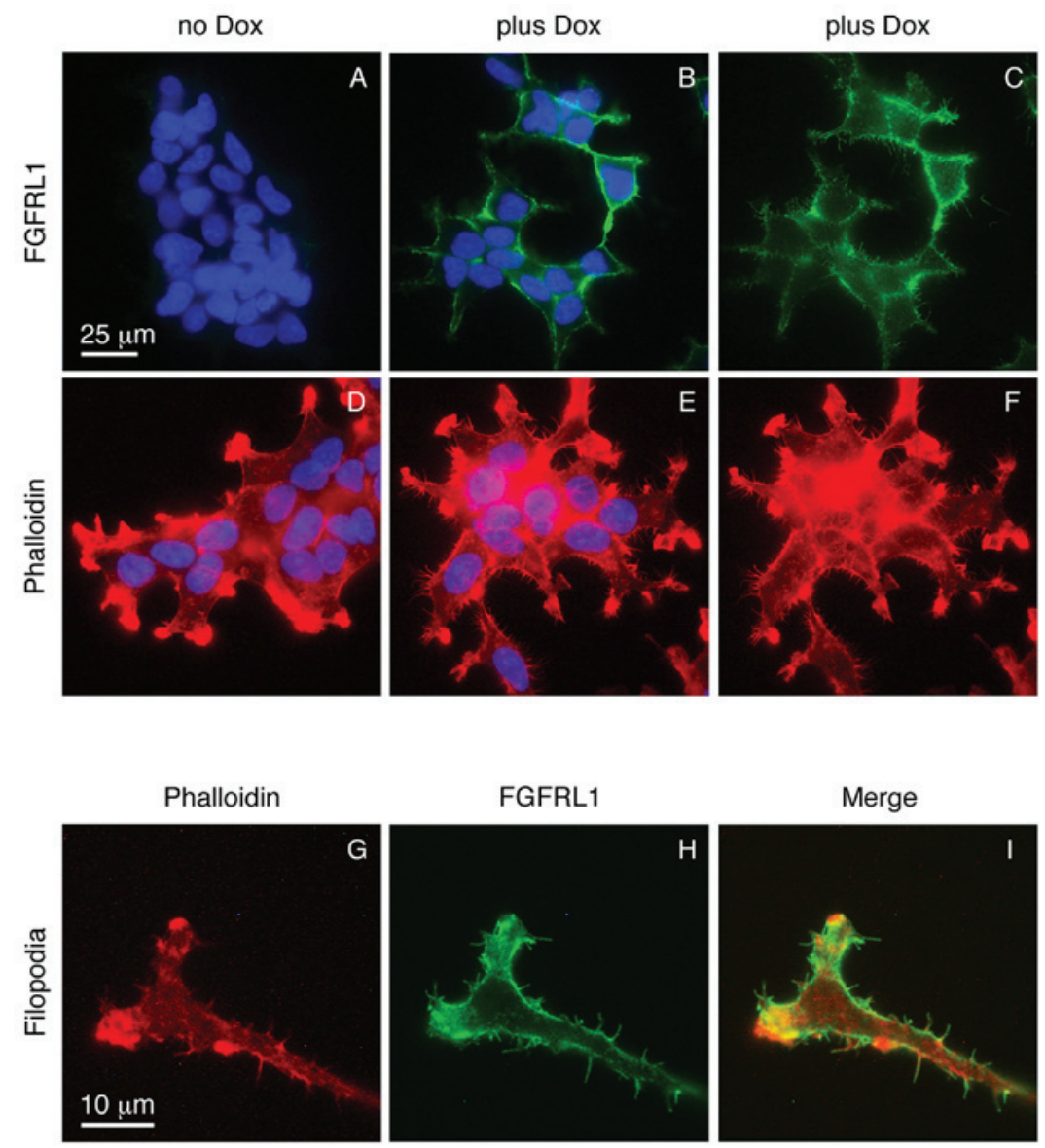

Figure 1. Expression of FGFRL1 in HEK 293 Tet-On-FGFRL1 $\Delta$ C cells induces the formation of filopodia-like spikes. FGFRL1-inducible cells (clone K13 $\Delta$ C) were cultivated in the absence (no Dox) or presence (plus Dox) of the inducer doxycycline. (A-C) Expression of FGFRL1 $\Delta \mathrm{C}$ was visualized with a monoclonal antibody against human FGFRL1, followed by Cy2-labeled secondary antibodies (green). FGFRL1 $\triangle \mathrm{C}$ was observed primarily at the cell membrane and in microspikes that emerged from the cell membrane. (D-F) Filamentous actin was stained with tetramethylrhodamine-labeled phalloidin (red). (G-I) Merging of the pictures stained for FGFRL1 and for actin indicated that the filopodia-like spikes partially colocalized with filamentous actin. In the absence of doxycycline, no FGFRL1 $\triangle$ C expression was observed and the number of filopodia-like spikes was much lower. For easy reference, cell nuclei stained with 4',6-diamidino-2-phenylindole are included in panels A, B, D, and E. FGFRL1, fibroblast growth factor receptor-like 1; Dox, doxycycline.

Nude mice xenograft experiments. To investigate tumorigenicity, immunodeficient $\mathrm{CD}-1{ }^{\circledR} \mathrm{NU} / \mathrm{NU}-\mathrm{Foxn} 1$ nude mice (Charles River Wiga GmbH, Sulzfeld, Germany) were utilized. Approximately $1 \times 10^{7} \mathrm{~K} 13 \Delta \mathrm{C}$ cells in PBS were subcutaneously injected at two ventral sites into the animals. Half of the mice (4 mice per group, randomly selected) were treated with $100 \mu \mathrm{g} / \mathrm{ml}$ doxycycline, which was directly added to the drinking water of the animals; the other half received regular water. After 5 weeks, the mice were sacrificed and images of the tumors were captured. All animal experiments had been approved by the Ethics committee of the County of Bern.

Statistical analysis. The significance of the results from the xenograft experiments was analyzed with the exact Fisher test utilizing an online calculation tool (http://www. quantitativeskills.com). $\mathrm{P} \leq 0.05$ was considered to indicate a statistically significant difference.

\section{Results}

FGFRL1 overexpression in cell culture. All the following experiments were performed with clone $\mathrm{K} 13 \Delta \mathrm{C}$ from the Tet-On-FGFRL1 $\Delta \mathrm{C}$ cell line (17). This clone had been prepared with cDNA for FGFRL1, which covered the extracellular domain and the transmembrane helix of the protein, but lacked the intracellular domain. Hence, all effects observed with this clone may be attributed to the extracellular and the transmembrane domains of FGFRL1.

In the absence of doxycycline, the $\mathrm{K} 13 \Delta \mathrm{C}$ clone did not express any detectable FGFRL1 $\Delta \mathrm{C}$ protein, as verified with our monoclonal antibodies (Fig. 1A). Following induction with doxycycline, a strong signal for FGFRL1 was observed at the cell membrane (Fig. 1B). Thus, K13 $\Delta \mathrm{C}$ cells tightly controlled the expression of FGFRL1 $\Delta \mathrm{C}$ depending on the presence or absence of doxycycline. When the focus of the microscope lens was changed to the plane of the glass slide (rather than the site of strongest fluorescence), some fluorescent signal was observed at numerous protrusions that emerged from the plasma membrane of the cells in a spike-like fashion (Fig. 1C). To investigate whether these microspikes represented normal filopodia, the cells were stained with fluorescently labeled phalloidin, which is known to interact with filamentous actin. Again, a number of spikes was observed that protruded in a perpendicular manner from the plasma membrane (Fig. 1D-F). At higher magnification (objective lens, 100X), the FGFRL1 $\Delta$ C signal (green) partially 


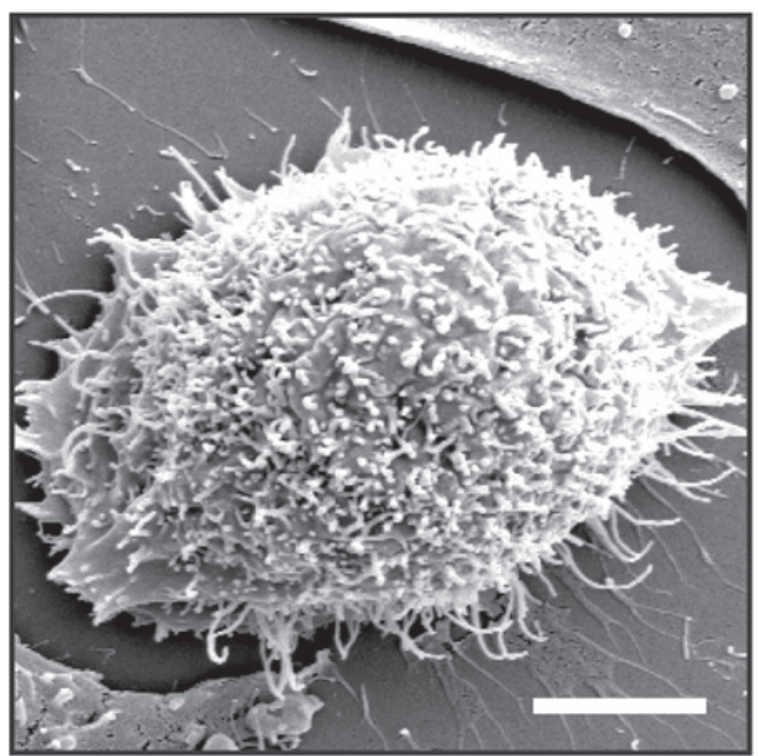

Figure 2. Electron microscopy of HEK 293 Tet-On-FGFRL1 $\Delta$ C cells. Clone $\mathrm{K} 13 \Delta \mathrm{C}$ was cultivated in the presence of doxycycline, fixed with glutaraldehyde and prepared for SEM. Inspection by SEM at $10 \mathrm{kV}$ detected numerous filopodia-like spikes with a diameter of $\sim 200 \mathrm{~nm}$. Bar, $5 \mu \mathrm{m}$. SEM, scanning electron microscopy.

co-localized with the phalloidin signal (red) (Fig. 1G-I). Additionally, the spikes were visualized under a scanning electron microscope (Fig. 2). Here, numerous protrusions were detected that had an average diameter of $180 \pm 28 \mathrm{~nm}$ $(n=6)$. It is therefore likely that the spikes represent regular filopodia. Notably, a much larger number of filopodia was observed in the presence of doxycycline compared with the number observed in its absence (Fig. 1D-F). Thus, forced expression of FGFRL1 $\triangle \mathrm{C}$ appears to stimulate the formation of filopodia.

Next, it was investigated whether $\mathrm{K} 13 \Delta \mathrm{C}$ cells would bind to purified FGFRL1 protein, as has previously been documented in Chinese hamster ovary (CHO) cells (15). The surface of a plastic plate (non-tissue culture) was coated with droplets of recombinant FGFRL1 solution comprising the $\operatorname{Ig} 1-\operatorname{Ig} 3$ domains, or with droplets of BSA that served as a control. Within $1 \mathrm{~h}$, the $\mathrm{K} 13 \Delta \mathrm{C}$ cells attached to the recombinant FGFRL1 protein, however, they did not attach to the control BSA (Fig. 3). It is likely that this interaction was accomplished by the binding of FGFRL1 to cell surface heparan sulfate proteoglycans, since the binding could be blocked with soluble heparin (data not shown), as previously reported in CHO cells (15).

In the following experiment, the effect of doxycycline (and therefore the effect of FGFRL1 $\Delta$ C) on cell-cell adhesion and cell clustering was recapitulated (Fig. 4). K13 $\Delta \mathrm{C}$ cells formed large patches of 10-30 cells following $9 \mathrm{~h}$ of incubation on bacterial plates in the presence of doxycycline. In the absence of the inducer, the cells also started to form clusters, however, these clusters were much smaller. After 3 days in culture, the $\mathrm{K} 13 \Delta \mathrm{C}$ cells had merged to form a continuous network of cells. Notably, the borders between the individual cells were no longer distinguishable in the presence of doxycycline, whereas they were clearly detectable in its
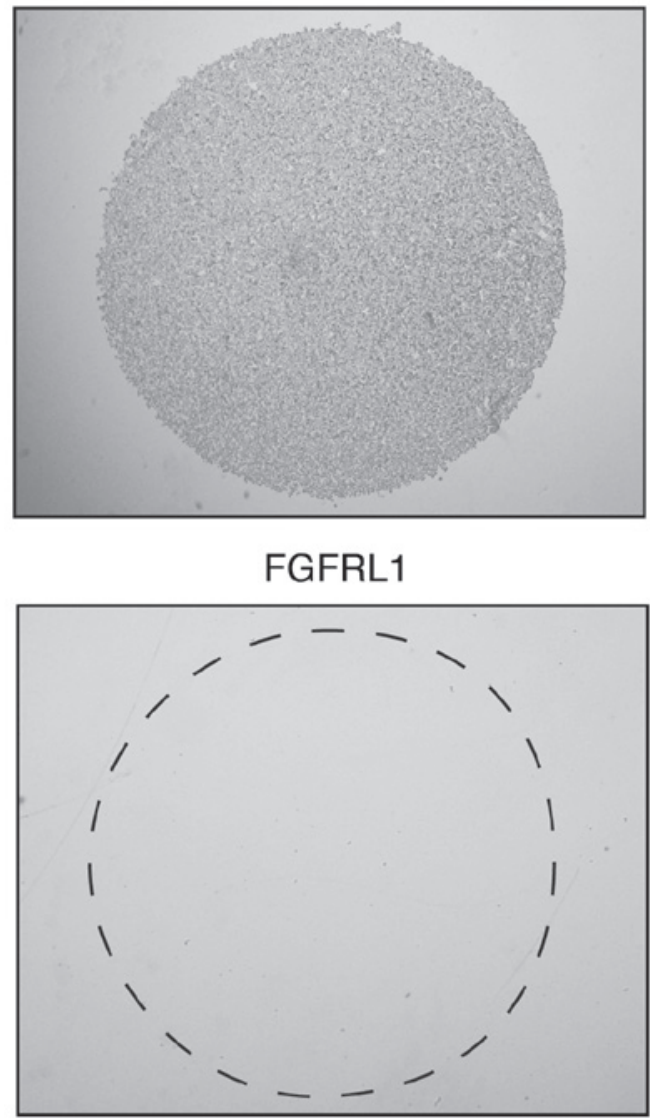

BSA

Figure 3. FGFRL1 induces cell adhesion. Droplets of a solution of recombinant FGFRL1 (or of BSA, which acted as a control) were spotted onto bacterial petri dishes and incubated overnight at $4^{\circ} \mathrm{C}$ in a humidified chamber. Residual binding sites of the plastic surface were blocked with BSA. HEK 293 Tet-On-FGFRL1 $\Delta$ C cells (clone K13 $\Delta$ C) were seeded onto the pre-coated dishes and allowed to attach for $1 \mathrm{~h}$. Non-adherent cells were washed off; adherent cells were fixed. FGFRL1 promoted cell attachment, whereas BSA (indicated by the dotted circle) did not. FGFRL1, fibroblast growth factor receptor-like 1; BSA, bovine serum albumin.

absence. Thus, the presence of FGFRL1 $\Delta \mathrm{C}$ promotes tight, intimate interactions between cells. Taken together, the three experiments suggested that FGFRL1 represents a typical cell adhesion protein.

Xenograft tumor model. The majority of cell adhesion proteins can function as tumor suppressors $(2,3)$; therefore, the effects of FGFRL1 on tumor formation were investigated. $\mathrm{K} 13 \Delta \mathrm{C}$ cells were injected subcutaneously into immunocompromised nude mice at two ventral sites. Half of the mice received doxycycline in their drinking water in order to induce the expression of FGFRL1 $\triangle \mathrm{C}$. The other half served as controls and received regular drinking water. Within 5 weeks, the control animals had developed large tumors (diameter $\geq 12 \mathrm{~mm}$ ) at five out of the eight injection sites (Fig. 5). In sharp contrast, none of the doxycycline-treated mice had produced any tumors $(\mathrm{P}=0.0257)$. A control experiment confirmed that doxycycline itself had no effect on tumor growth (data not shown). Thus, FGFRL1 may function as a typical tumor suppressor that effectively inhibits the outgrowth of xenografted tumors in vivo. 

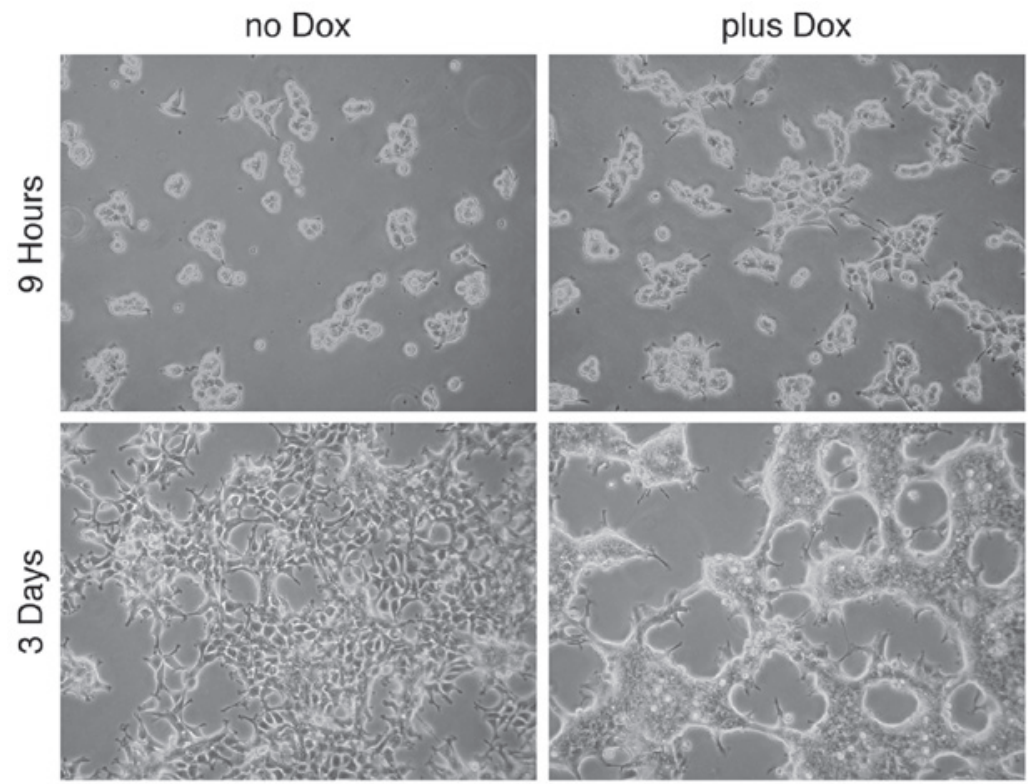

Figure 4. FGFRL1 induces cell-cell adhesion and cell clustering. FGFRL1-inducible HEK 293 Tet-On cells (clone K13 $\Delta$ C) were seeded into bacterial petri dishes and cultivated for up to 3 days in the absence (no Dox) or presence (plus Dox) of doxycycline. Expression of FGFRL1 $\Delta$ C induced cell-cell adhesion and cell clustering. After 3 days, the boundaries between cells were barely detectable in the presence of doxycycline, whereas they were clearly detectable in its absence. FGFRL1, fibroblast growth factor receptor-like 1; Dox, doxycycline.

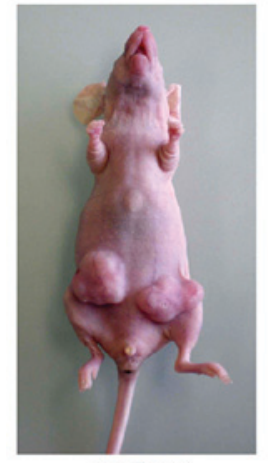

no Dox

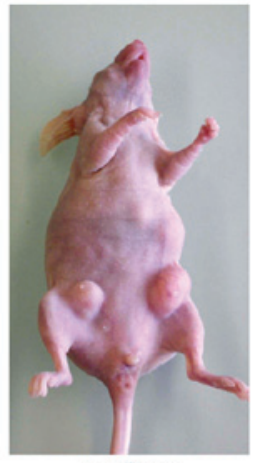

no Dox

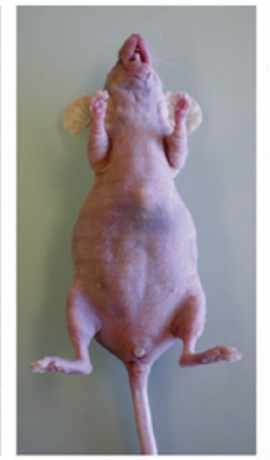

plus Dox

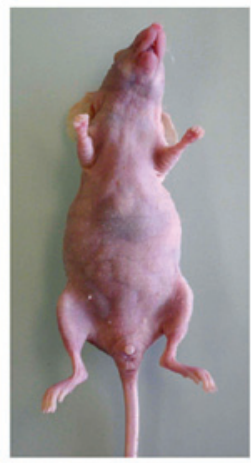

plus Dox

Figure 5. FGFRL1 acts as a tumor suppressor in vivo. Inducible HEK 293 Tet-On-FGFRL1 $\Delta$ C cells (clone K13 $\Delta$ C) were injected into nude mice at two ventral positions. Expression of FGFRL1 was induced in half of the animals by doxycycline, which was added to their drinking water. The other half received normal drinking water. Tumor formation was inspected at the injection sites after 5 weeks. Two representative examples of each group are depicted. The injected cells were able to generate tumors only in those mice that did not receive any doxycycline. FGFRL1, fibroblast growth factor receptor-like 1; Dox, doxycycline.

\section{Discussion}

The current study, taken together with results from the literature, demonstrates that the novel receptor FGFRL1 represents a regular cell-cell adhesion protein. This conclusion is based on the following facts: i) The domain structure of FGFRL1, which has a single transmembrane domain, three extracellular Ig-like repeats and a short intracellular domain (21), resembles the structure of other cell adhesion proteins from the Ig superfamily, namely the nectins and nectin-like molecules $(22,23)$. ii) Recombinant polypeptides corresponding to the extracellular domain of FGFRL1 promote cell adhesion in vitro. If a mutation is introduced into the polypeptide chain, the activity is completely lost (15). iii) When overexpressed in different cell lines, FGFRL1 protein accumulates at intersections where two cells touch each other (15). Overexpression in HEK 293 Tet-On cells leads to the aggregation of the cells and to the formation of large clusters (17). iv) Finally, the results of the current study indicate that FGFRL1, like other typical cell adhesion proteins, acts as a tumor suppressor in a xenograft tumor model. It was determined that the forced expression of FGFRL1 in HEK 293 Tet-On cells completely inhibited the outgrowth of tumors in immunocompromised mice.

During the xenograft experiments, it was noted that the cells were not extremely tumorigenic. A large number of HEK 293 Tet-On-FGFRL1 $\Delta$ C cells had to be injected into the mice to initiate any tumor growth. Other studies have also noted that HEK 293 cells exhibit particularly low tumorigenicity. Shen et al (24) demonstrated that HEK 293 cell tumorigenicity increased with increasing passage number, and finally reached $100 \%$ when the passage number was $>65$; however, the original 
isolates of the cells did not form tumors at all in nude mice. In the present study, an outgrowth of tumors was detected in the absence of doxycycline in 5 cases (63\%). Following induction of FGFRL1 synthesis, tumor growth was observed in no cases $(0 \%)$. Doxycycline itself had no effect on tumor growth, as demonstrated in a control experiment and as previously published in the literature (25). Thus, it is FGFRL1 that can act as a tumor suppressor.

Previous studies have reported that alterations in the synthesis of FGFRL1 occur in tumor cells (26-31). The screening of 241 different human tumor samples with a cancer-profiling array suggested that major changes in the relative expression of FGFRL1 occur in ovarian tumors (26). In several samples, a significant decrease in FGFRL1 expression was observed in the tumor tissue relative to the matched control tissue. However, in one ovarian tumor sample there was a 25-fold increase (26). Furthermore, the overexpression of FGFRL1 in certain ovarian tumor samples was confirmed in a study aiming to identify novel tumor-specific marker genes (27). An association of FGFRL1 expression with tumor growth and metastasis was also suggested by a study of patients suffering from head and neck tumors $(28,29)$. In this case, FGFRL1 overexpression appeared to correlate with tumor growth. Another study noted a significant decrease of FGFRL1 protein expression in bladder tumors and bladder cancer cell lines (30). This decrease was explained by heterozygous deletions at the chromosomal region 4p16.3, which includes the locus of the FGFRL1 gene. Finally, mutations affecting the reading frame of FGFRL1 have been observed relatively frequently in colorectal cancer cell lines (31). The majority of these mutations occurred in the intracellular domain of the protein.

The molecular mechanism governing how FGFRL1 may inhibit tumor growth in a xenograft model is complex. With other cell adhesion proteins it has been concluded that forced expression partially restores contact inhibition of the tumor cells. In the case of CADM1, it was demonstrated that homophilic interactions of CADM1 at the surface of two adjacent cells activated the phosphatidylinositol 3-kinase (PI3K) pathway and led to the reorganization of the actin cytoskeleton (32). The intracellular domain of CADM1 formed a complex with membrane-associated guanylate kinase homologues, including MPP3 and Dlg, thus linking CADM1 and PI3K. In the case of FGFRL1, the effect may be exerted by heterophilic interactions of the extracellular domain of FGFRL1 with another transmembrane protein, since the protein expressed by $\mathrm{K} 13 \Delta \mathrm{C}$ cells lacks the intracellular domain of FGFRL1. The extracellular domain has the ability to interact with target proteins of neighboring cells containing heparan sulfate chains, including syndecans and glypicans (15). Two target proteins, glypican-4 and glypican-6, which specifically interact with FGFRL1, have previously been identified by our group using tandem LC mass spectrometry (16). Therefore, it is hypothesized that FGFRL1 inhibited tumor growth in the current study in the following manner: HEK 293 Tet-On cells lost their normal contact inhibition and grew in an unrestricted way, as they were subcloned multiple times during the generation of the HEK 293 Tet-On-FGFRL1 $\Delta$ C clones. When injected into nude mice, these cells gave rise to large tumors due to a defect in contact inhibition. Enforcing the expression of FGFRL1 $\Delta \mathrm{C}$ in the injected cells by adding doxycycline induced the adhesion of the cells with each other and restored contact inhibition, thereby inhibiting tumor growth.

It remains to be demonstrated whether the identification of FGFRL1 as a tumor suppressor may be exploited to improve the diagnosis and therapy of cancer patients. FGFRL1 may serve as a tumor marker to identify aggressive tumors that have lost FGFRL1 expression (27,31). Furthermore, there may be the possibility of enhancing FGFRL1 synthesis in the tumor tissue. Levels of FGFRL1 mRNA are regulated by microRNA-120 $(28,33)$, which specifically interacts with the 3 ' end of the FGFRL1 mRNA and leads to its degradation. Therefore, if it were possible to downregulate microRNA-120 expression in the tumor tissue, the endogenous levels of FGFRL1 would increase and contact inhibition of the cells in the tumor tissue would be restored, thus suppressing tumor growth. Future studies are necessary to determine whether this could be developed as a novel therapeutic strategy for patients with cancer.

In conclusion, the current study taken together with results from the literature, demonstrates that FGFRL1 is a cell-cell adhesion protein that acts as a tumor suppressor similar to numerous other cell adhesion proteins.

\section{Acknowledgements}

The current study was supported by grants from the Swiss National Science Foundation (Bern, Switzerland; no. 31003A-143350), the Novartis Foundation for Medical-Biological Research (Basel, Switzerland; no. 15A006) and the Swiss Foundation for Research on muscular diseases (Cortaillod, Switzerland; no. 2015-12-18).

\section{References}

1. Kreis $\mathrm{T}$ and Vale R (eds): Guidebook to the Extracellular Matrix, Anchor and Adhesion Proteins, 2nd editon. Oxford University Press, 1999.

2. Okegawa T, Li Y, Pong RC and Hsieh JT: Cell adhesion proteins as tumor suppressors. J Urol 167: 1836-1843, 2002.

3. Moh MC and Shen S: The roles of cell adhesion molecules in tumor suppression and cell migration: A new paradox. Cell Adh Migr 3: 334-336, 2009.

4. Pećina-Slaus N: Tumor suppressor gene E-cadherin and its role in normal and malignant cells. Cancer Cell Int 3: 17, 2003.

5. Ren B, Yu YP, Tseng GC, Wu C, Chen K, Rao UN, Nelson J, Michalopoulos GK and Luo JH: Analysis of integrin alpha7 mutations in prostate cancer, liver cancer, glioblastoma multiforme, and leiomyosarcoma. J Natl Cancer Inst 99: 868-880, 2007.

6. Heller G, Geradts J, Ziegler B, Newsham I, Filipits M, Markis-Ritzinger EM, Kandioler D, Berger W, Stiglbauer W, Depisch D, et al: Downregulation of TSLC1 and DAL-1 expression occurs frequently in breast cancer. Breast Cancer Res Treat 103: 283-291, 2007.

7. Ito T, Shimada Y, Hashimoto Y, Kaganoi J, Kan T, Watanabe G, Murakami Y and Imamura M: Involvement of TSLC1 in progression of esophageal squamous cell carcinoma. Cancer Res 63: 6320-6326, 2003.

8. Wiedemann $\mathrm{M}$ and Trueb B: Characterization of a novel protein (FGFRL1) from human cartilage related to FGF receptors. Genomics 69: 275-279, 2000.

9. Trueb B: Biology of FGFRL1, the fifth fibroblast growth factor receptor. Cell Mol Life Sci 68: 951-964, 2010.

10. Trueb B, Zhuang L, Taeschler S and Wiedemann M: Characterization of FGFRL1, a novel fibroblast growth factor (FGF) receptor preferentially expressed in skeletal tissues. J Biol Chem 278: 33857-33865, 2003.

11. Ornitz DM and Itoh N: The fibroblast growth factor signaling pathway. Wiley Interdiscip Rev Dev Biol 4: 215-266, 2015. 
12. Gerber SD, Steinberg F, Beyeler M, Villiger PM and Trueb B: The murine Fgfrll receptor is essential for the development of the metanephric kidney. Dev Biol 335: 106-119, 2009.

13. Baertschi S, Zhuang L and Trueb B: Mice with a targeted disruption of the Fgfrl1 gene die at birth due to alterations in the diaphragm. FEBS J 274: 6241-6253, 2007.

14. Amann R, Wyder S, Slavotinek AM and Trueb B: The FgfrL1 receptor is required for development of slow muscle fibers. Dev Biol 394, 228-241, 2014.

15. Rieckmann T, Kotevic I and Trueb B: The cell surface receptor FGFRL1 forms constitutive dimers that promote cell adhesion. Exp Cell Res 314: 1071-1081, 2008.

16. Steinberg F, Gerber SD, Rieckmann T and Trueb B: Rapid fusion and syncytium formation of heterologous cells upon expression of the FGFRL1 receptor. J Biol Chem 285 37704-37715, 2010

17. Yang X, Steinberg F, Zhuang L, Bessey R and Trueb B: Receptor FGFRL1 does not promote cell proliferation but induces cell adhesion. Int J Mol Med 38: 30-38, 2016.

18. Zhuang L, Gerber SD, Kuchen S, Villiger PM and Trueb B Deletion of exon 8 from the EXT1 gene causes multiple osteochondromas (MO) in a family with three affected members Springerplus 5: 71, 2016.

19. Rieckmann T, Zhuang L, Flück CE and Trueb B: Characterization of the first FGFRL1 mutation identified in a craniosynostosis patient. Biochim Biophys Acta 1792: 112-121, 2009.

20. Henning A, Schneider M, Bur M, Blank F, Gehr P and Lehr CM: Embryonic chicken trachea as a new in vitro model for the investigation of mucociliary particle clearance in the airways. AAPS PharmSciTech 9: 521-527, 2008.

21. Zhuang L, Falquet L and Trueb B: Genome-wide comparison of FGFRL1 with structurally related surface receptors. Exp Ther Med 1: 161-168, 2010

22. Mandai K, Rikitake Y, Mori M and Takai Y: Nectins and nectin-like molecules in development and disease. Curr Top Dev Biol 112: 197-231, 2015.

23. Samanta D and Almo SC: Nectin family of cell-adhesion molecules: Structural and molecular aspects of function and specificity. Cell Mol Life Sci 72: 645-658, 2015.
24. Shen C, Gu M, Song C, Miao L, Hu L, Liang D and Zheng C: The tumorigenicity diversification in human embryonic kidney 293 cell line cultured in vitro. Biologicals 36: 263-268, 2008.

25. Tanaka $\mathrm{T}$ and Rabbitts $\mathrm{TH}$ : Interfering with RAS-effector protein interactions prevent RAS-dependent tumour initiation and causes stop-start control of cancer growth. Oncogene 29: 6064-6070, 2010.

26. Schild C and Trueb: Aberrant expression of FGFRL1, a novel FGF receptor, in ovarian tumors. Int J Mol Med 16: 1169-1173, 2005.

27. Barrett CL, DeBoever C, Jepsen K, Saenz CC, Carson DA and Frazer KA: Systematic transcriptome analysis reveals tumor-specific isoforms for ovarian cancer diagnosis and therapy. Proc Natl Acad Sci USA 112: E3050-E3057, 2015.

28. Tsuchiya S, Fujiwara T, Sato F, Shimada Y, Tanaka E, Sakai Y, Shimizu K and Tsujimoto G: MicroRNA-210 regulates cancer cell proliferation through targeting fibroblast growth factor receptor-like 1 (FGFRL1). J Biol Chem 286: 420-428, 2011.

29. Shimada Y, Okumura T, Nagata T, Hashimoto I, Sawada S, Yoshida T, Fukuoka J, Shimizu K and Tsukada K: Expression analysis of fibroblast growth factor receptor-like 1 (FGFRL1) in esophageal squamous cell carcinoma. Esophagus 11: 48-53, 2014.

30. di Martino E, Taylor CF, Roulson JA and Knowles MA: An integrated genomic, transcriptional and protein investigation of FGFRL1 as a putative 4p16.3 deletion target in bladder cancer. Genes Chromosomes Cancer 52: 860-871, 2013.

31. Donnard E, Asprino PF, Correa BR, Bettoni F, Koyama FC, Navarro FC, Perez RO, Mariadason J, Sieber OM, Strausberg RL, et al: Mutational analysis of genes coding for cell surface proteins in colorectal cancer cell lines reveal novel altered pathways, druggable mutations and mutated epitopes for targeted therapy. Oncotarget 5: 9199-9213, 2014.

32. Murakami S, Sakurai-Yageta M, Maruyama T and Murakami Y: Trans-homophilic interaction of CADM1 activates PI3K by forming a complex with MAGuK-family proteins MPP3 and Dlg. PLoS One 9: e110062, 2014.

33. Huang X, Ding L, Bennewith KL, Tong RT, Welford SM, Ang KK, Story M, Le QT and Giaccia AJ: Hypoxia-inducible mir-210 regulates normoxic gene expression involved in tumor initiation. Mol Cell 35: 856-867, 2009. 